\title{
Arthritis
Research UK
}

\section{Mechanisms of chronic pain and fatique}

\section{PARTICIPANT INFORMATION SHEET Version 2 09/06/2017}

IRAS Ref No.: 207362

We would like to invite you to take part in a research study exploring mechanisms of chronic pain and fatigue. Before you decide it is important for you to understand why the research is being done and what it will involve.

- Please take time to read the following information carefully and discuss it with others if you wish.

- Please ask us if there is anything that is not clear or if you would like more information.

Part 1 explains the purpose of the study and what will happen to you if you take part.

Part 2 gives you more detailed information about the conduct of the study.

This project is collaboration between Brighton and Sussex University Hospitals NHS Trust and Brighton and Sussex Medical School.

Please remember your decision whether to take part or not will not affect your current or future care in any way.

\section{PART 1}

\section{What is the purpose of the study?}

We wish to understand further the biological reasons why people with Fibromyalgia and/or ME/Chronic Fatigue Syndrome experience chronic pain and/or fatigue. We know, , that people who experience Fibromyalgia not only suffer with muscle and joint pain, but also of physical and mental fatigue, often called 'fibro fog'. People who experience chronic fatigue, such as those with ME/Chronic Fatigue Syndrome also suffer with mental fatigue and can experience pain. We know that these experiences can often have a considerable impact on quality of life and yet at present are often poorly understood. We know that in such conditions there may be abnormalities in the 'fight or flight' involuntary nervous system and blood tests may also show more markers of inflammation (the way the body reacts to infection, irritation or other injury).

This project seeks to understand in detail how abnormalities in these bodily responses affect each other and interact to cause pain and fatigue. We will recruit people who experience mainly pain, those who experience mainly fatigue and those who experience both and people who experience neither We will test for differences between groups and relate these to symptoms.

Firstly, we will measure how pain and fatigue change when the flight and fight nervous system is activated by lying in a tilted position. Secondly, we will examine effects of inducing a mild state of inflammation in the body using brain scanning to measure differences in brain structure and function (with blood markers) between people with pain and/or fatigue, and healthy people.

We hope to better understand the relationship between the brain and the body in these conditions and hope that the findings will help inform new treatment approaches. 
Why have I been invited to take part?

You have been invited to take part as you either experience Fibromyalgia or ME/Chronic Fatigue Syndrome or both conditions.

In order to take part in the study should be able to lie comfortable for about an hour. You should not have received a Typhoid vaccine in the last three years or have previously experienced any adverse responses to Typhoid vaccination. You should also not have had any other vaccinations or have taken part in a Clinical Trial of an Investigational medicinal Products (CTIMP) in the last 6 months. Pregnancy is an exclusion criteria for the study.

Do I have to take part?

No. It is up to you to decide whether or not to take part. If you then decide to take part you are still free to withdraw at any time, without needing to give a reason.

Study overview

\section{Questions about you}

- Medical history

- Fibromyalgia symptoms

- ME/Chronic Fatigue symptoms

- Questionnaires about pain, fatigue, mood and attention

- Blood tests and pressure sensitivity measurement

- Heart rate and blood pressure measurements

- Tilt table test including measurements of pain, fatigue, mood and attention

- Hair sample

- Questionnaires about pain, fatigue, mood and attention

- Blood tests

- Injection

- Tasks and questionnaires

- Brain scan

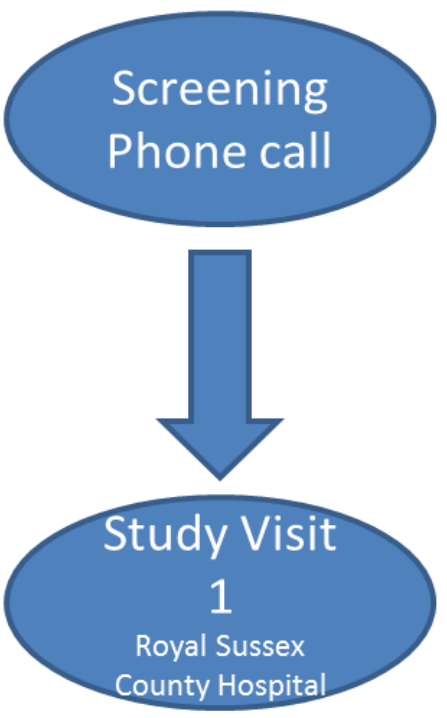

$15-45$ mins

$2-3$ hours $£ 30$ reimbursement

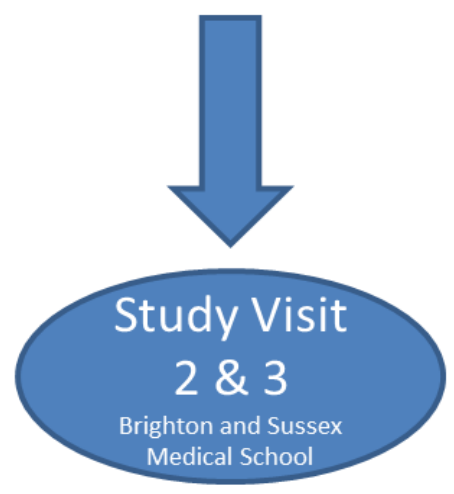

4 hours each visit £30 reimbursement each visit 
What will happen to me if I take part?

Please see below.

\section{Detail of the study}

If you are interested in taking part you will be invited to have a telephone screening conversation to see firstly if you are suitable to take part in the study and secondly which group of the study you will be part of. This involves making sure you are suitable for the study (e.g. are you ok to have blood tests, to lie flat and also safe to go in the brain scanner?) and asking standard questions about your medical history and condition(s), e.g. about your pain and fatigue. The answers to these questions will be kept strictly confidential. If you are suitable for the study and willing to take part we will then invite you to the study visits.

The study involves three visits on separate occasions. Each visit lasts between 2.5 and 4 hours and will include breaks and an opportunity to stretch. The first study visit takes place at the Clinical Investigation and Research Unit at Royal Sussex County Hospital, Brighton and involves heart rate and blood pressure measurements. The other two study visits take place at the Clinical Imaging Sciences Centre at Brighton and Sussex Medical School at The University of Sussex, Falmer and involves going in the brain scanner. All study visits also involve blood tests, questionnaires and performing some simple tasks which will be explained in more detail later in the leaflet.

On each the days that you come we will ask you not to have any caffeine or nicotine for 2 hours prior to the study. If you are taking medication one of the researchers will contact you to discuss whether you should also refrain from taking your medication until after the study. This is because caffeine, nicotine and some medications can affect your heart rate or blood pressure and thus the results of some of the tests. However, you will be under no obligation to refrain from taking medication.

Prior to the first study visit you will be asked to complete some questionnaires about your symptoms. You will be free to take as long as you need to do so, but we do not expect that they would take longer than one hour. This can be done offline via paper questionnaires or online.

Study Visit 1 - Clinical Research Facility (CIRU), Royal Sussex County Hospital, Brighton

- You will be met by a researcher and the study visit will be explained to you. The researcher will go through a consent form with you and will be asked to sign this if you are happy to take part.

- Then you will fill out some brief questionnaires about your current symptoms and we will examine your joints. We will also gently measure how sensitive you are to physical pressure and take some blood. These are blood tests routinely carried out by your doctor and include kidney and thyroid function and functioning of the immune system. 


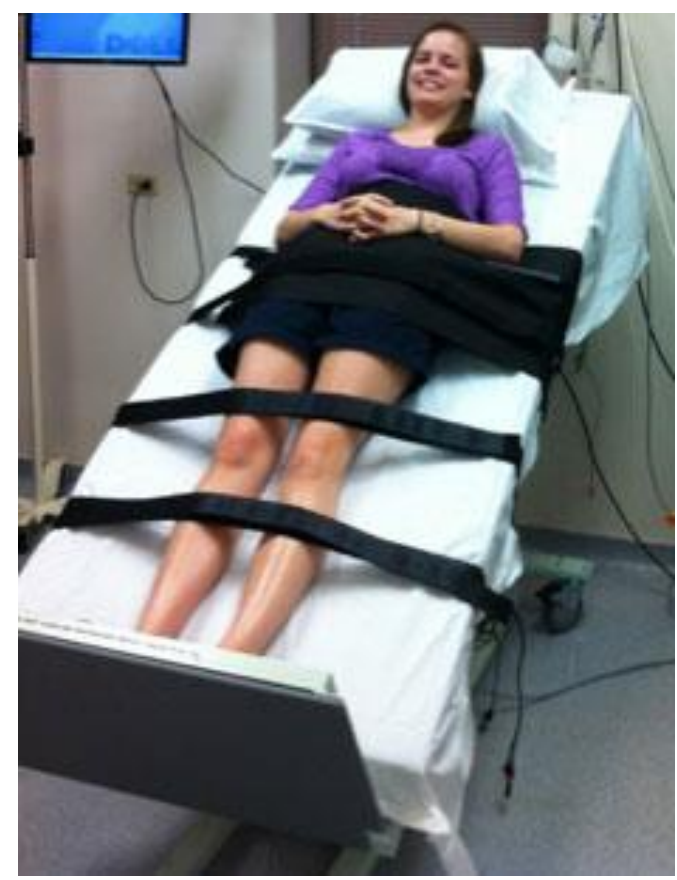

We are interested in measuring the responses of the involuntary nervous system and do so by attaching a cuff to your finger to measure heart rate and blood pressure continuously whilst you to undertake some simple exercises like breathing fast and slow, gripping hard and counting backwards. We ask you to do this whilst lying flat on something called a tilt table, to which you are securely strapped. We are interested to see the responses in the body to the difference between lying and standing and how this might affect pain and fatigue and for this reason we will then place you securely in a tilted position, as illustrated in this picture. Placing people on a tilt table is frequently done in clinical practice.

When you are in a tilted position we will then measure levels of pain and fatigue and sensitivity to physical pressure, all the while measuring your heart rate and blood pressure. We will also ask you questions about your symptoms and asks you to do some short tasks. You will be in the tilted position for about 20 minutes. If you feel uncomfortable at any point we can bring you back to a lying position. We will then return you to the flat position and detach the equipment.

Finally, we will take a small sample of your hair to look for long term sings of activation of the "fight and flight" nervous system. Fibromyalgia and ME/Chronic Fatigue syndrome have been associated with an altered response to stress and a way to measure this is by studying the levels of the stress hormone, cortisol, in your hair. We will take 2-3 small strands of your hair at the back of the head with a diameter of approximately half of a pencil. We will cut the hair as close as possible to the scalp as you can see in the image below. We will only collect samples once and the whole procedure will be painless. Hair samples will be stored in a locked cupboard at CRF/CIRU prior to being sent to Kingston University for Analysis.
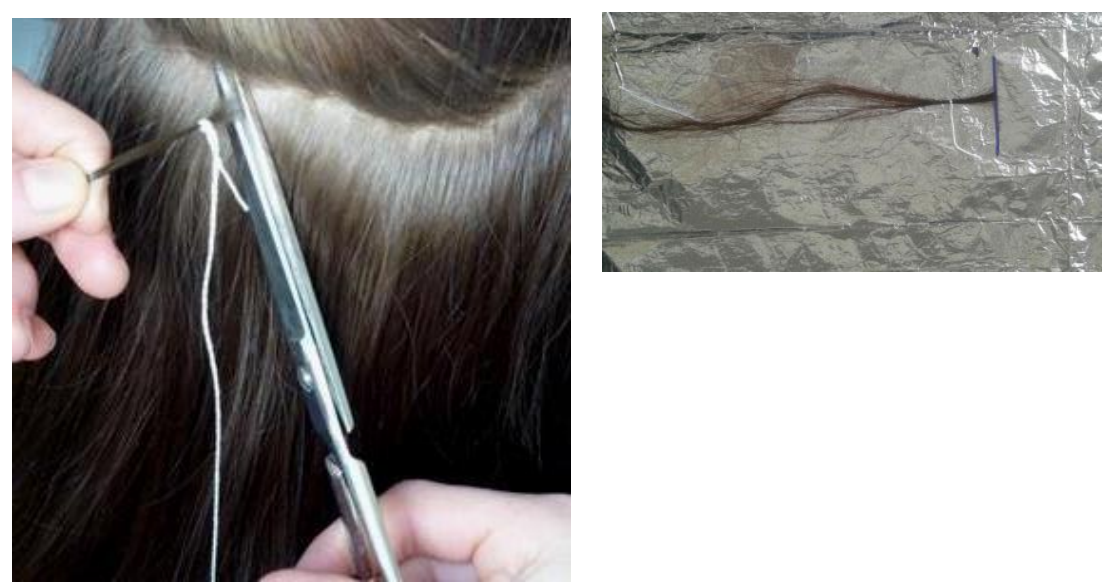
This will be the end of the visit and you will be reimbursed $£ 30$.

\section{Study Visit 2 and 3 - Clinical Imaging Sciences Centre, University of Sussex, Falmer}

- When you arrive for the second study visit one of our team members will meet you at the reception desk and you will have another opportunity to ask any further questions.

- If you are happy to continue with the study we will then ask you to fill in a consent form.

- We will take some baseline measurements of heart rate and blood pressure whilst lying down and standing up (no tilt table involved) and breathing deeply. We will measure how sensitive you are to physical pressure.

- We will then take some blood to look at levels of inflammation in the blood and then then ask you to complete some short questionnaires about your symptoms

- A qualified doctor will give you the injection of typhoid (Typhim Vi) vaccine or placebo (saline injection) into the deltoid muscle of your upper arm. Although the doctor will know whether you received the typhoid vaccine or the saline injection neither you nor the other researchers will be told. This is to prevent knowledge of which injection you were given influencing your or the researcher's behaviour. We will then ask you to rest whilst the injection takes effect.

- During this time you will be free to read a book or newspaper, though we would ask that you remain within the centre. We will then take some further measurements of heart rate and blood pressure whilst lying down and standing up (no tilt table involved) and breathing deeply.

- We will measure how sensitive you are to physical pressure.

- We will then ask you to complete some short questions about your symptoms

- We will then take another sample of blood. This will include levels of inflammation and also markers of gene activity. This is not a genetic test.

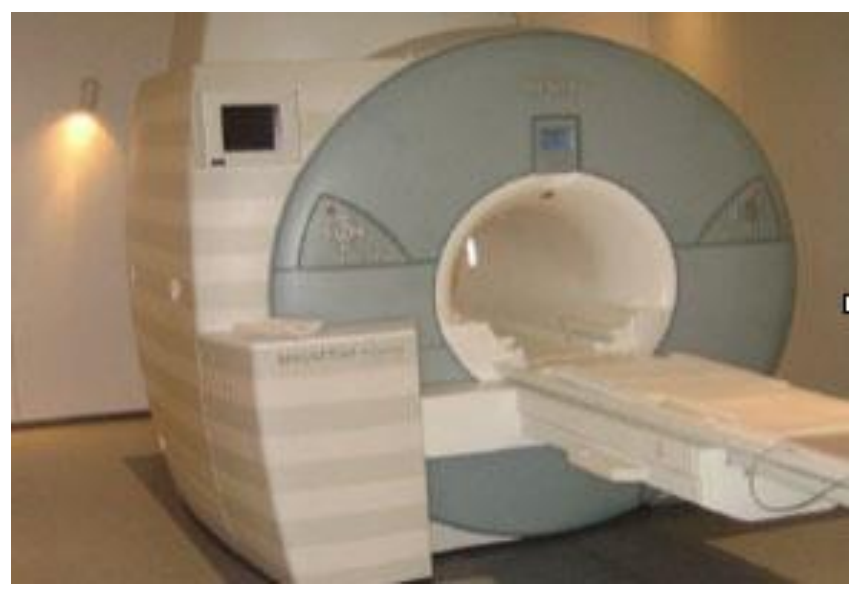

You will then be taken through to the MRI scanner. The MRI scanner is a short tube with a strong magnet inside. You will be asked to remove any metal items that you may be wearing e.g. rings, piercings, watch, necklace and glasses and if needed given a pair of MRI safe glasses to wear. The MRI scanner is very noisy so will also give you earplugs and headphones to wear. We will ask you to lie still in the scanner while we take some images of the structure of your brain and assess how your brain responds to the injection you were given. We will also ask you to perform some tasks in the scanner related to attention and also to emotion processing whereby you look at pictures and words and make responses using a button box. In total you will be in the scanner for about an hour. You will have the opportunity to stretch in the scanner between tasks. When you come out of the scanner this will be the end of the visit. You 
will have the opportunity to ask questions and will reimbursed $£ 30$.

On the third study visit one of our team members will meet you at the reception desk. Exactly the same procedure will be followed as your second visit, only you will be given typhoid vaccine or placebo depending on the injection that you were given during your previous visit. Again, you will not know which you received on each occasion until you have completed your participation.

Visits 2 and 3 will happen after visit 1 and will be approx. 2 weeks apart.

\section{Storage of blood samples}

Blood samples collected at visit one will be sent to the Pathology Lab at the Royal Sussex County hospital for analysis and processing. Blood samples collected at visits 2 and 3 will be processed and stored (frozen at $-80^{\circ} \mathrm{C}$ ) at Brighton and Sussex Medical School, until they are analysed at an academic laboratory within the UK. All samples will be anonymized.

\section{Expenses and payments}

You will be paid £30 for each study visit so $£ 90$ for the three study visits.

\section{What are the possible risks in taking part?}

There are no substantial risks to taking part. All procedures (blood sampling, tilt table, typhoid vaccination injection, and MRI brain scanning) are safe and routinely used in clinical care, however it is important to understand how we ensure your safety throughout the study.

At all study visits a medical doctor will be on site at all times. At each study visit blood will be taken. This will be done by an experienced clinician. Other than possible mild bruising and discomfort there are no other particular risks.

In study visit 1, when you are placed in the tilted position, there is a small possibility that you might faint if you have ever or nearly fainted before. You will be in a hospital with clinical staff and equipment to manage this if this happens.

In either study visit 2 or 3 you will receive a Typhoid vaccination. The vaccination used in this study is a routinely administered typhoid vaccine that is given to people who work in or travel to parts of the world where typhoid is common. It is a mild inflammatory stimulus and serious side effects are extremely rare. However, like any medication, there is a small risk of an allergic reaction (anaphylaxis). A medically qualified doctor will therefore be present throughout the study. Minor swelling and discomfort/pain around the injection site or headaches may occur up to 72 hours after administration. These effects are short lasting and usually resolve without any treatment. If you feel unwell or experience any other side effects following injection you are advised to contact your GP or nearest A\&E department telling them they you have recently had a typhoid vaccination.

In both study visit 2 and 3 you will have a MRI brain scan. MRI is a safe procedure that has been used for many years without any particular problems being found. It does not involve xrays or other forms of radiation. Some participants may experience dizziness or a slight tingling feeling (due to the fast changes of magnetic fields that occur during an MRI scan) or claustrophobia due to being inside of the scanner. While you are in the scanner you will have a 
button that you can press and you will also be able to communicate over an intercom, so if you should feel uncomfortable in the scanner you can alert the radiographer and the scan can be stopped.

With regards to your personal data, information from the study will be protected and anonymous, so that people will not have access to the information about who took part or find out results.

\section{What are the possible benefits of taking part?}

There are no immediate benefits to you of taking part in this study. However you will receive a free Typhoid vaccination which may be useful if you are planning to travel to a foreign country with poor sanitation.

Although this research may not directly benefit you, it could result in better understanding of the biological basis of chronic pain and fatigue and help understand Fibromyalgia and ME/Chronic Fatigue Syndrome and inform new treatment approaches

\section{What mechanisms are in place if my answers to questionnaires or tasks suggest my current health is of particular concern?}

It is possible that your answers to some of the questionnaires or tasks may indicate that your symptoms are worse than usual. The research doctors are trained clinicians and if this is the case we will contact your doctor to inform them of this.

\section{Will my taking part in this study be kept confidential?}

Yes. With the exception of the above scenario we want to emphasise that all results obtained from the study will be strictly confidential and will only be used for research purposes. Further details of how this information will be stored are detailed in part 2 below.

What if there is a problem?

Any complaint about the way you have been dealt with during the study or any possible harm you might suffer will be addressed. The detailed information on this is given in part 2.

\section{PART 2}

\section{What will happen if I don't want to carry on with the study?}

You may withdraw at any point during the study. This will not affect your future care in any way.

\section{What if there is a problem?}

If you have a concern about any aspect of this study, you should ask to speak to the researchers (Prof Kevin Davies +44 (0)1273 877890, Dr Jessica Eccles +44 (0)1273 873833 or Dr Kristy Themelis +44 (0)1273 873121) in the first instance, who will do their best to answer your questions. If you remain unhappy and wish to complain formally, you can do this either by contacting the Research and Development department at Brighton and Sussex University Hospitals Trust (01273 696955) or the Patient Advice and Liaison Service at Brighton and Sussex University Hospitals (01273 696955). 
Will my taking part in the study be kept confidential?

Yes. All the information about your participation in this study will be secured against any unauthorised access. Although the overall results will be published in medical journals, no individual subjects will be identifiable from this.

\section{Involvement of the General Practitioner (GP)}

Your GP will not routinely be notified that you have taken part in this study. However we will ask if we can contact your GP to advise if the results of your tests suggest any problems that require further management.

\section{What will happen to the results of the research study?}

The recordings of bodily responses and questionnaires will be anonymised (removed of identifying information) and kept in locked offices at CIRU at Royal Sussex County Hospital and at Brighton and Sussex Medical School.

The results of the questionnaires, along with all other information collected from you in the course of this research will be kept strictly confidential. The results will be statistically analysed and findings subsequently published in peer reviewed journals. You will not be identified in any publications. You are more than welcome to receive a summary of the results of this work which can be obtained by giving us your email or postal address.

You will be given the opportunity to consent for our research team to store the anonymized brain images you provide for further analysis in this study and another ethically approved study on fatigue (IRAS: 201462).

\section{Who has funded this study?}

This study is funded by a grant from Arthritis Research UK (AR UK)

\section{Who has reviewed the study?}

All research in the NHS is looked at by independent group of people, called a Research Ethics Committee, to protect your interests.

\section{What are the indemnity arrangements?}

The University of Sussex holds insurance policies which apply to this study. If you experienced harm as a result of your participation in this study, you may be able to claim compensation. Please contact one of the researchers (Prof Kevin Davies +44 (0)1273 877890, Dr Jessica Eccles +44 (0)1273 873833) in case you would like further information about the insurance arrangements which apply to this study.

\section{Contact for further Information}

Many thanks for reading this. We hope you feel able to take part in our study. If you are interested in taking part or have any questions, please contact Dr Kristy Themelis for further information on k.themelis@bsms.ac.uk or +44 (0)1273 873121. 